\title{
(C) OPEN ACCESS \\ Empowering junior doctors: a qualitative study of a QI programme in South West England
}

\author{
Natasha J Doran, ${ }^{1,2,3}$ Rob Bethune, ${ }_{1}^{1,4}$ Joanne Watson, ${ }^{5}$ Katherine Finucane, ${ }_{1}^{6}$ \\ Andrew Carson-Stevens ${ }^{7}$
}

'South West Academic Health Science Network, Exeter, UK ${ }^{2}$ Medical School, University of Exeter, Exeter, UK

${ }^{3}$ Department for Health, University of Bath, Bath, UK

${ }^{4}$ Royal Devon and Exeter NHS Foundation Trust, Exeter, UK

${ }^{5}$ Torbay and South Devon NHS Foundation Trust, Torquay, UK ${ }^{6}$ North Bristol NHS Trust, Bristol, UK

${ }^{7}$ Division of Population Medicine, School of Medicine, Cardiff University, Cardiff, UK

Correspondence to Natasha J Doran, South West Academic Health Science Network, Exeter EX2 5AZ, UK n.doran@exeter.ac.uk

Received 10 August 2018 Revised 17 October 2018 Accepted 20 October 2018 Published Online First 13 November 2018

\begin{abstract}
Aim To explore how the South-West Foundation Doctor Quality Improvement programme affected foundation year 1 (F1) doctors' attitudes and ability to implement change in healthcare.

Methods Twenty-two qualitative interviews were carried out with two cohorts of doctors. The first F1 group before and after their participation in the QI programme; the second group comprised those who had completed the programme between 1 and 5 years earlier. Qualitative data were analysed using thematic analysis techniques.
\end{abstract}

Results Prior to taking part in the QI programme, junior doctors' attitudes towards QI were mixed. Although there was agreement on the importance of QI in terms of patient safety, not all shared enthusiasm for engaging in QI, while some were sceptical that they could bring about any change. Following participation in the programme, attitudes towards QI and the ability to effect change were significantly transformed. Whether their projects were considered a success or not, all juniors reported that they valued the skills learnt and the overall experience they gained through carrying out QI projects. Participants reported feeling more empowered in their role as junior doctors, with several describing how they felt 'listened to' and able to 'have a voice', that they were beginning to see things 'at systems level' and learning to 'engage more critically' in their working environment.

Conclusions Junior doctors are ideally placed to engage in QI. Training in QI at the start of their medical careers may enable a new generation of doctors to acquire the skills necessary to improve patient safety and quality of care.

\section{BACKGROUND}

Following key reports by Francis, ${ }^{1}$ Berwick $^{2}$ and Keogh, ${ }^{3}$ quality improvement (QI) interventions have been gaining in popularity, while coming under increasing scrutiny within the discourse of improvement science. ${ }^{4-7}$ Improvement science is currently emerging as a new discipline ${ }^{89}$ requiring cross-disciplinary research between the clinical and social sciences, as well as more collaboration between practitioners and researchers.

The development of educational programmes designed to use the skills, knowledge and enthusiasm of clinicians and other frontline staff to engage in QI work is beginning to emerge ${ }^{10} 11$; however, to date, much improvement work has been viewed as 'peripheral'- a mere 'add on' to one's day job. ${ }^{12}$ In order to meet the vision laid down in the Berwick report, ${ }^{2}$ those working on the front line of complex healthcare systems need protected time to access the training and resources to engage in and lead QI. ${ }^{12}$ Building time to train in QI at the start of one's medical career may help to develop a new workforce with the resilience and improvement skills necessary to meet these growing challenges.

Most medical student undergraduate curricula do not include teaching and training related to QI. ${ }^{13}{ }^{14}$ Although the involvement of clinicians in collaborative improvement projects is becoming increasingly sought after, training in QI remains variable. ${ }^{14}$ Yet junior doctors encounter many of the problems affecting quality and their front-line position and number make them a potentially huge force for driving improvement. ${ }^{1011} 131516$ A number of initiatives have been conducted to involve junior doctors in QI with significant success. ${ }^{17-20}$ The South-West Foundation Doctor Quality Improvement (SWFD QI) programme supports foundation year 1 (F1) doctors in groups of 6-10, to identify, design and carry out a QI project relating to their clinical practice. Core skills and techniques drawn from QI methodology are taught at meetings, while emphasis is placed on the experiential learning gained through carrying out QI projects.

Set up in August 2010, the programme (previously known as the Severn Deanery Foundation Doctor QI programme) ${ }^{11}$ now involves 10 hospitals, 100 QI projects and over 1000 doctors. $^{21-30}$ In order to understand whether junior doctors feel able and indeed want to implement change in healthcare, as well as determine the overall impact of the programme, we designed this qualitative study to explore junior doctors' perceptions and experience of the SWFDQI programme.

\section{METHODS}

We undertook a qualitative longitudinal study involving 22 interviews with 15 F1 doctors now working in a number of locations across the UK (see box 1).

To capture any change in attitude towards QI, seven F1s were interviewed twice, prior to and following their participation in the programme (2014-2015). Eight doctors were also interviewed who had completed the QI programme between 1 and 5 years prior (historical group).

Interviewees were self-selecting volunteers who were purposefully sampled (in terms of being about to embark on, or having prior experience of the QI programme) in order to gain access to information-rich cases ${ }^{31}$; however, by incorporating 


\section{Box 1 Interview sample}

\section{Doctors:}

- Female: 9.

- Male: 6.

- Age range 22-37.

Participants were located in the following geographical areas at interview:

Taunton ( $n=3)$, Yeovil $(n=4)$, Cardiff $(n=1)$, London $(n=2)$, Australia ( $n=2)$, Bath $(n=1)$, Swindon $(n=1)$ and Gloucester $(n=1)$.

the views of the before and after group of F1 volunteers with an historical group, we also adopted maximum variation in our sampling strategy.

Participants were invited to take part via email which included information sheets and consent forms. Interviews were based on a topic guide (see box 2) and lasted between $30 \mathrm{~min}$ and $50 \mathrm{~min}$.

Interviews were recorded, transcribed and checked to exclude identifying information. Fieldwork notes were kept to contextualise the data and detailed summaries of interviews were produced. Thematic analysis techniques were used to generate a description of themes both within and across the dataset. ${ }^{32}$ The phases of analysis included coding, followed by the identification and clustering of themes and subthemes and the production of a descriptive summary. Team members then met to discuss the analysis, after which the themes and subthemes were grouped to construct a more interpretative narrative across the dataset and depicted diagrammatically (see figure 1 ).

\section{RESULTS}

The main theme 'changing the culture of practice' is discussed in relation to the following sub-themes: initial perception of QI; professional role, junior status and National Health Service (NHS) hierarchy; barriers to QI and successful outcomes; and changing attitudes and perceptions.

\section{Initial perception of QI}

Most participants felt that QI should be part of their job. However, prior to taking part in the QI programme, some expressed the view that they would prefer to focus on more 'clinical stuff' and that it was not their 'personal goal to be a change trendsetter' (QIVP3)

At initial interview, although many acknowledged the need for QI, some felt frustrated regarding time constraints and conscious of their junior status:

\section{Box 2 Interview topic guide}

- Interviewees were asked about their experience of the quality improvement (QI) programme and their projects.

- They were asked what they had learnt in relation to QI and whether they felt the programme had enabled them to implement change in practice.

- Further questions were aimed at probing their attitude to implementing change in healthcare and whether the programme affected the way they saw their professional role.

- They were also asked whether they experienced any challenges during their projects and whether they could identify areas for improvement with the QI programme.
I feel like it's everyone's role to raise issues [...], otherwise we're just not going to move forward as a trust or as a whole NHS. But [...] I don't want to go round sort of saying, 'we should do this,' when it's probably already being thought of by someone more senior. (QIVP6)

Prior to taking part in the QI programme, some participants were sceptical about their ability to engage in any meaningful change:

Inevitably, realistically a lot of them [problems in hospital system] can't be changed [...] I don't know, sometimes I just feel like what's the point? This isn't going to change. (QIVP1)

Others felt that they would need more support and team engagement and even then their option for change might be blocked:

We'd need a lot more support than just a junior trying to make changes in the NHS. And I think that's just from being on the wards and seeing how hard it is. (QIVP6)

As junior doctors, the prospect of making any changes within such a complex organisation felt daunting:

It [instigating change] can be daunting and you often think 'well I'm here on my own, I'm just one junior so no one's going to listen to me. (QIVP4)

The pressures inherent in adjusting to their new working environment meant that many were initially of the view that making any changes to the processes and systems of care was beyond their capability, remit and role.

\section{Professional role, junior status and NHS hierarchy}

Participants described a change in perception of their professional role, as the responsibilities extended to them through engaging in the QI programme brought them into contact with more senior levels within the NHS hierarchy.

One thing that's been really helpful is you get to meet people that you would never have met before, [...] as a junior doctor. I mean [...] I could quite easily have access to people quite high up in the organisation. (QIH4)

Having support from seniors and particularly management support, was felt to provide more legitimacy for their improvement projects:

Having one of the associate medical directors as a mentor, and being able to email them and call them by their first name and talk through projects with them, that's suddenly a very kind of normalising thing [...], so then, I guess it's more empowering, as a Junior. (QIH4)

Participants described how QI work enabled them to 'have a voice' (QIH2) and to feel more 'valued' and 'listened to' by their seniors (QIH3). Rather than feeling conscious of their junior status, participants began to appreciate how best they might contribute to the wider system, given their unique position and role:

I think that $[\ldots]$ having fresh eyes on things $[\ldots]$ means that we [F1s] probably see things differently to people who've been there a really long time and you get desensitised to things that are wrong or could be improved. (QIVP1)

As junior doctors you sometimes see things that senior clinicians don't have to engage with, so they miss. (QIVP4b)

Through the shared goal of improving the processes and systems of care for patients, participants reportedly felt more empowered in their role as newly qualified doctors, as it led 


\section{Turning the Tanker}

Engaging Junior Doctors in Quality Improvement

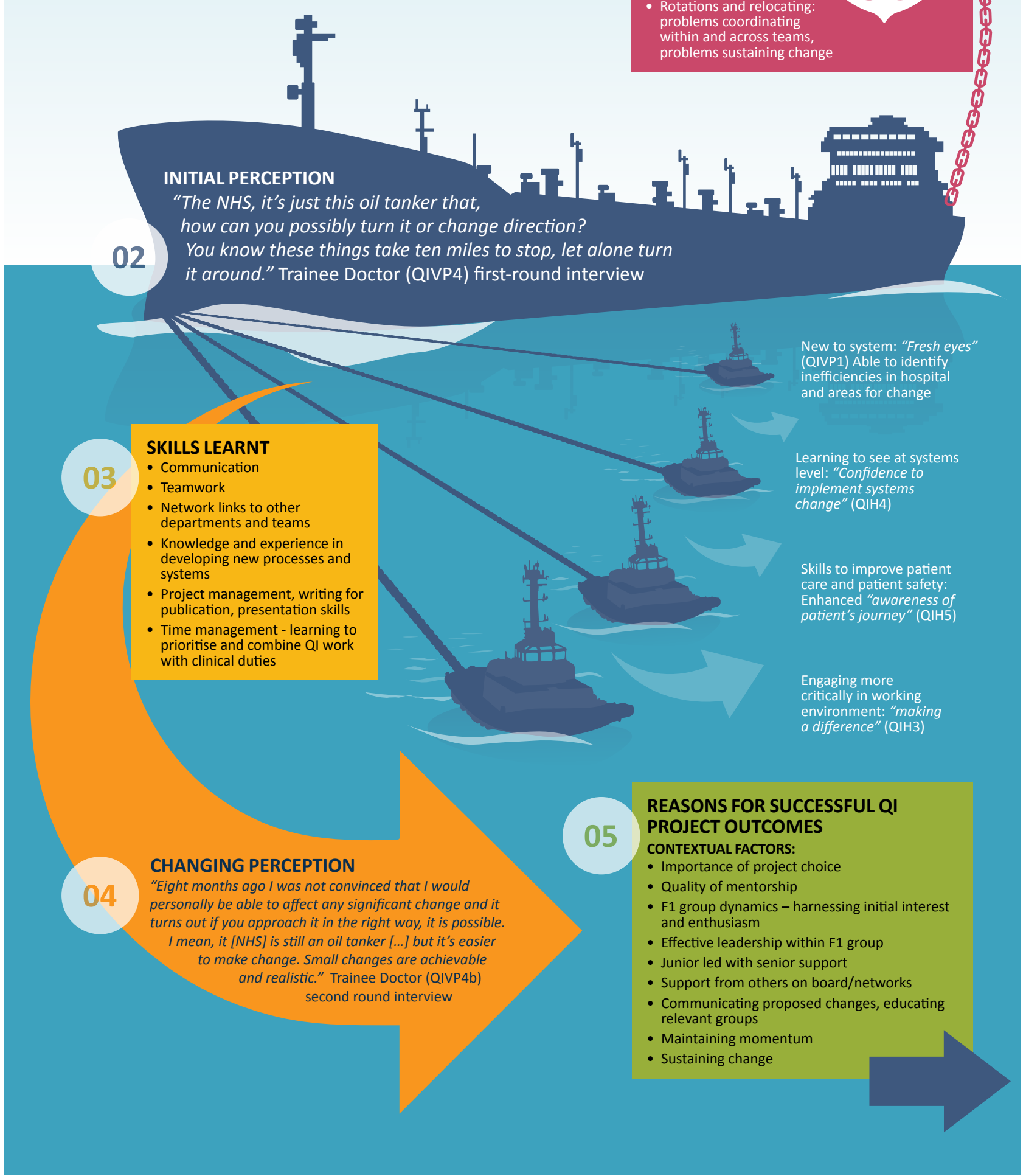

Figure 1 Turning the tanker: engaging junior doctors in quality improvement. 
them to engage with all levels of the system and to develop an increased awareness of their responsibility to identify and instigate change.

\section{Barriers to QI and successful outcomes}

Most participants described experiencing times of frustration in their attempts to bring about change:

Well I think if I talk about the initial experiences where we got knocked back you know, you become frustrated and disillusioned with change. (QIVP6b)

In particular, encountering resistance from seniors or other teams and departments was a challenge:

At the beginning they seemed really quite resistant to it [QI]. I think talking to people and getting them to see what you're working towards can be quite a challenge to begin with (QIH3)

It's hard as an F1,... not to necessarily do what your Reg [senior] says, because they're the one who's in charge and you're not, really. So even if you say, 'Well no, it's because of this project and it's been shown to be safe,' they'd be like, 'Well I don't really care, this is what I want'. (QIVP3b)

For several participants, trying to coordinate their proposed change across teams proved frustrating:

It's difficult when there are lots of people and they're all doing different things, to coordinate, to get everything to work together. (QIVP3b)

Freeing up time to engage in QI and to follow through on agreed commitments was also seen as an obstacle within timestretched services:

The main barrier would be people being busy, and not being able to do the things which they said, and they were trying to be able to do. (QIVP3b)

For many participants, rotations and moving locations proved a barrier to QI work:

The one thing that's difficult when you're a doctor in the UK, because you're moving from hospital to hospital on a six month to yearly basis, $[\ldots]$ the difficulty arises from making sure that change continues when you're not working there. (QIH2)

In some cases, encountering challenges strengthened resolve and project outcome:

At the start we had difficulty implementing it with some of the senior nursing staff. We had real trouble with the GP group, but $[\ldots]$ they've [obstacles] probably been advantageous to the project, because it's just more solid because of that, because we've addressed the problems rather than painting over the top of them. (QIVP7b)

In those instances where projects were viewed to be less successful, this was still considered to be a worthwhile learning experience:

I'm probably better equipped now [...] because I've done things which have worked and I've done things that haven't worked.

(QIH2)

Participants who reported having a positive experience of QI, particularly where their project was seen to be a success or where they were able to implement a more sustained change, were more likely to want to engage in future QI. Those in the historical group who reported benefiting from inspired leadership during their F1 year felt more positive about QI despite encountering obstacles to this type of work in other hospital settings. Encountering obstacles such as moving every 6-12 months, lack

\section{Box 3 Example quality improvement projects}

- Improving the safety and timeliness of warfarin prescription. ${ }^{24}$

- Improving the safety of weekend handover on hospital wards. $^{25}$

- Standardising the common clinical equipment junior doctors need on surgical wards. ${ }^{27}$

- Improving access to trust guidelines for junior doctors. ${ }^{28}$

- Safer intravenous fluid prescribing. ${ }^{29}$

of senior support, lack of time and other clinical priorities were to some extent mitigated by such positive mentorship.

Participants identified a number of contextual factors that they felt were important for successful project outcomes (see figure 1). These included: the importance of project choice (see box 3); quality of mentorship; F1 group dynamics to include effective leadership and the importance of harnessing initial enthusiasm; to be junior led with senior support; network links to engage system-wide support; educating and communicating proposed changes to relevant groups; maintaining momentum; and having strategies in place to sustain change.

Overall, the importance of time, teamwork and effective communication were seen as vital ingredients to enhance project outcome.

\section{Changing attitudes and perceptions}

Having completed the QI programme and their project work, F1s' attitudes towards QI and their perception of the hospital system could be seen to change:

As an F1 there's so much going on and you're just anxious and scared about starting that, you know, those bigger problems and bigger ideas are kind of in the periphery, but yes, certainly I feel they are targets which are much more in my line of sight now. (QIVP7b)

After carrying out their QI projects, participants reported feeling more confident to suggest areas for change and more equipped to improve patient care through identifying inefficiencies in their hospital system:

There's a huge potential to improve systems and improve patient care as a result of that, which I wasn't really aware of before. (QIVP7b)

I think it's definitely given me a bit of confidence in thinking, if you see something day in and day out that's going wrong or you can improve it [...], it just wouldn't make sense for you not to even let someone know. Or think, I can take that on and make a change there. (QIH3)

Several participants also described a change in their perception of their role and responsibilities as they learnt to engage more critically in their working environments:

It has changed my perspective of things [...], as well as being a doctor and the sort of day-to-day, that there is this extra need for us to try and help change and $[. .$.$] to question things more. It has made$ me realise that change is needed. (QIVP6b)

The QI programme was also thought to enhance their awareness of the patient's experience:

It [QI programme] raised an awareness of the patient's journey. We were given a lot of [...] personal stories from patients' relatives or patients themselves. I think that made a big difference. (QIH5) 
For many participants, making a difference to patient care and patient safety through their QI projects impacted positively on their self-confidence and self-belief:

I think lots of junior doctors, they don't feel like they can make a difference, but what was evident from our QI project is that you can, and if you put the work into it, then it can make a big difference to patient safety. (QIH6)

The longer term impact of the QI programme was reflected by the historical group. All members of this group felt it had had a positive impact on their career progression and that the skills they learnt (see figure 1) were of ongoing benefit:

It [QI programme] enabled me to get new knowledge at that level, $[\ldots]$ to carry it forward and learn things that work and don't work. (QIH2)

I now feel confident to do quality improvement projects wherever I go, so I plan to do one when I'm in a GP practice, it will be valuable for the practice and for patients. (QIH6)

Following participation in the QI programme, all participants came to the realisation that implementing systems change was achievable and worthwhile. Having initially felt disillusioned with change (see figure 1), participants reported seeing value in making changes, however small, to their working environment:

You don't necessarily have to change the world, just your little bit [...], these things add up over time, and especially in this current culture $[\ldots]$ I think that's the thing, is to keep chipping away at it. (QIVP4b)

Even if it's something small, done by one person, you can make big changes. And it's not always easy to change a culture, but the more attention is paid to it, then the more can be changed. (QIH5)

Through being introduced to QI work at an early stage of their careers, participants were able to envisage the longer term potential of this learning and its application to culture change:

I think culture is really generational [...] if our generation can implement things which we carry through, then when we're of the generation that the leaders of the hospital are now, you know, the culture will have changed. It's difficult to get appreciation for that if you look at just a short timescale. (QIVP7b)

\section{DISCUSSION}

These findings highlight the changing views and attitudes towards QI among a cohort of junior doctors and a group of more senior doctors reflecting back to their F1 year. Examples of changing the culture of working practice were evident in that participants reportedly felt listened to, were able to impact changes to their working environment and, as a consequence, their attitude towards QI, their understanding of the wider hospital system and their perception of their professional role could be seen to change.

There is a paucity of research about the benefits of involving junior doctors in QI at this stage of their careers, as opposed to medical students, or more experienced doctors. ${ }^{33}$ Findings from this qualitative study suggest participation by F1s in a QI project overrides initial scepticism about the value of QI in healthcare. Seeing improvements for patients raises their self-confidence and self-belief that they can lead change and empowers them in their role to provide and improve care. F1s also experience resistance to change first hand and recognise reasons for successful project outcomes. Overall, F1s identified QI as achievable and worthwhile for patients and themselves.

Resistance from staff towards change processes in healthcare has been well documented in the literature. ${ }^{34-36}$ This study demonstrates how encountering resistance and finding ways to negotiate, adapt and work through such barriers provides F1s with invaluable experiential knowledge or 'metis'. ${ }^{37}$ Through the SWFDQI programme, ${ }^{11}$ F1s were not simply learning a new methodological approach, but were learning through trial and error, how context, engagement, adaptation and sustainability were key to the success of any QI project. Such factors have been recognised as central to the planning and implementation of QI in healthcare. ${ }^{14}$

The SWFDQI programme aims to facilitate and support a co-operation structure among junior doctors which, given time, may promote new social norms in working practice through a programme of shared learning and collaboration. To understand contextual factors either aiding or hindering successful implementation of improvement initiatives in localised settings, more longitudinal qualitative studies are required, particularly ethnographic studies. ${ }^{38-40}$ Additionally, more formative, theory-driven evaluations of comparative educational programmes for health professionals are needed that can describe both the content and successful execution strategies. ${ }^{71}$ Efforts to report on patient and system outcomes are also needed to realise the impact of these educational programmes on practice. Future programmes would benefit from adopting a 'relay' approach between cohorts of participants, as opposed to 'start and stop' projects.

Sharing the learning generated by leading improvement projects is also important. As QI initiatives expand, and the current cohort of junior QI leaders become more senior, it is anticipated the focus of projects will become more advanced and ambitious. Informal educational communities, such as the Institute for Healthcare Improvement Open School, ${ }^{42}$ could make an important contribution to this emerging community of healthcare professionals learning to lead QI.

\section{Strengths and Limitations}

This longitudinal qualitative study provides an in-depth exploration of the experiences of a small cohort of doctors and their changing perceptions of engaging in QI during their F1 year. Participants were self-selecting volunteers who may have had strong views towards QI; however, triangulating the results of the before and after group of F1s who were new to the programme with the historical group, meant that interviewees represented a wide variation sample in terms of age, experience in practice and geographical location.

\section{CONCLUSION}

Given the right support structures, junior doctors can realise their unique role in QI in healthcare. Training in QI at the start of their medical careers enables a new generation of doctors to acquire the skills necessary to enhance patient safety and quality as their careers develop. Future evaluation should incorporate longitudinal designs to measure the impact of such educational

Main messages

- Junior doctors frontline position and number make them a potentially huge force for driving improvement

- Protected time is needed to access training and resources to engage in and lead quality improvement (QI).

- Training in QI at the start of one's medical career may help to develop a new workforce with the resilience and improvement skills to enhance patient safety and quality as their careers develop. 


\section{Current research questions}

To what extent can these qualitative findings be generalised to larger groups in other contexts?

- What impact are QI educational initiatives having on professional development, systems and patient outcomes?

- Considering the growing demand for QI across healthcare, what are the barriers and facilitators to the uptake of QI among other healthcare professional groups?

- What planning and resources are needed to robustly evaluate return of investment from QI training programmes?

\section{What is already known on the subject}

- QI interventions designed to bring about positive change to healthcare practice have been gaining in popularity

- Educational programmes to engage clinicians and other frontline staff in QI work are beginning to emerge.

- Much improvement work has been viewed as peripheral to one's day job.

- Training in QI remains variable.

initiatives on professional development, systems and patient outcomes. Comparable reporting of QI initiatives are needed to enable organisations to determine the benefit these programmes could have on patient care and experience.

Correction notice This article has been corrected since it was published Online First. The affiliation for Joanne Watson was incorrect and has now been corrected.

Acknowledgements On behalf of the South West Foundation Doctors Study Group, we would like to thank the following people who have made the programme work over the last 6 years: Patricia Woodhead, Eleanor Soo, Mark Dahill, Clare Van Hamel, Rachel Johns, Jo Howarth, Matt Hill and all of the junior doctors who have put so much enthusiasm into improving the care of the patients they serve. The authors would also like to thank all volunteers who kindly shared their experiences of the South-West Foundation Doctor Quality Improvement programme.

Contributors NJD cowrote the research proposal and contributed to the study design, the acquisition, analysis and interpretation of data and drafted the manuscript. RB cowrote the research proposal and secured funding and contributed to the study conception, design and interpretation of data; AC-S contributed to the study conception and design and interpretation of data. JW and KF secured funding and contributed to the study conception. All authors contributed to the critical revision of the paper and approved the final manuscript for publication.

Funding The study was funded by the South West Academic Health Science Network.

Disclaimer The study sponsor played no role in study design, data collection, analysis and interpretation, the writing of the article or the decision to submit it for publication.

Competing interests None declared.

Patient consent Not required.

Ethics approval According to the NHS Research Ethics Committee decision tool, ethical approval was not required for this study; however, informed consent was given with the anonymity of participants guaranteed.

Provenance and peer review Not commissioned; externally peer reviewed.

Data sharing statement Data generated or analysed during this study are included in this published article.

Open access This is an open access article distributed in accordance with the Creative Commons Attribution Non Commercial (CC BY-NC 4.0) license, which permits others to distribute, remix, adapt, build upon this work non-commercially, and license their derivative works on different terms, provided the original work is properly cited, appropriate credit is given, any changes made indicated, and the use is non-commercial. See: http://creativecommons.org/licenses/by-nc/4.0/

\section{REFERENCES}

1 Francis R. Report of the mid Staffordshire NHS foundation trust public inquiry, 2013.
2 Berwick D. National advisory group on the safety of patients in england. a promise to learn - a commitment to act. London: Department of Health, 2013.

3 Keogh B, Review into the quality of care and treatment provided by 14 hospital trusts in England: overview report NHS England. 2013. Available from: http://www.nhs.uk/ NHSEngland/bruce-keogh-review/Documents/outcomes/keogh-review-final-report.pdf [Accessed 02 May 2016].

4 Portela MC, Pronovost PJ, Woodcock T, et al. How to study improvement interventions: a brief overview of possible study types. BMJ Qual Saf 2015;24:325-36.

5 Wears RL. Improvement and evaluation. BMJ Qual Saf 2015;24:92-4.

6 Batalden P, Davidoff F, Marshall M, et al. So what? Now what? Exploring, understanding and using the epistemologies that inform the improvement of healthcare. BMJ Qual Saf 2011;20(Suppl 1):i99-i105.

7 Parry GJ, Carson-Stevens A, Luff DF, et al. Recommendations for evaluation of health care improvement initiatives. Acad Pediatr 2013;13(6 Suppl):S23-S30.

8 Marshall M, Pronovost P, Dixon-Woods M. Promotion of improvement as a science. The Lancet 2013;381:419-21.

9 Kuhn TS. The Structure of Scientific Revolutions. The University of Chicago press Ltd, 1962, 1996.

10 Lemer C, Moss F. Patient safety and junior doctors: are we missing the obvious? BMJ Qual Saf 2013;22:8-10

11 Bethune R, Soo E, Woodhead P, et al. Engaging all doctors in continuous quality improvement: a structured, supported programme for first-year doctors across a training deanery in England. BMJ Qual Saf 2013;22:613-7.

12 O'Malley H, Pereira P, Building Q. Learning from designing a large scale improvement community The Health Foundation, 2016. ISBN: 978-1-906461-79-9.

13 Dahill M, Bethune R, Carson-Stevens A, et al. First-year doctors' attitudes and beliefs relating to quality improvement and patient safety. Clin Risk 2015;21(2-3):47-9.

14 Quality Improvement - training for better outcomes. Academy of Medical Royal Colleges. 2016. Available from: www.aomrc.org.uk;

15 Neale G, Vincent C, Darzi SA. The problem of engaging hospital doctors in promoting safety and quality in clinical care. J $R$ Soc Promot Health 2007;127:87-94.

16 Carson-Stevens A, Patel E, Nutt SL, et al. The social movement drive: a role for junior doctors in healthcare reform. J $R$ Soc Med 2013;106:305-9.

17 Wong BM, Etchells EE, Kuper A, et al. Teaching quality improvement and patient safety to trainees: a systematic review. Acad Med 2010;85:1425-39.

18 Ahmed M, Arora S, Tiew S, et al. Building a safer foundation: the Lessons Learnt patient safety training programme. BMJ Qual Saf 2014;23:78-86.

19 Coltart CE, Cheung R, Ardolino A, et al. Leadership development for early career doctors. Lancet 2012:379:1847-9.

20 Henderson D, Carson-Stevens A, Bohnen J, et al. Check a Box. Save a Life: How student leadership is shaking up health care and driving a revolution in patient safety. J Patient Saf 2010;6:43-7

21 Stockdale C, Trivedi B, Jerome E, et al. Implementation of a combined Cardiopulmonary Resuscitation and Treatment Escalation Plan document in a District General Hospital. BMJ Qual Improv Rep 2014;2

22 Sneller S, Lada K, Turner C, et al. Improving the quality of weekend handover at Yeovil District Hospital. BMJ Qual Improv Rep 2014;3:u203647.w1613.

23 Lindley S, Robertson I. A standardised storage solution for venepuncture/cannulation equipment could save an NHS hospital the equivalent of a whole junior doctor. BMJ Qual Improv Rep 2014:2.

24 Dyar R, Hall S, McIntyre B. Warfarin prescription and administration: reducing the delay, improving the safety. BMJ Qual Improv Rep 2015:4:u204509.w1983.

25 Jardine AG, Page T, Bethune $R$, et al. Bring on the weekend - Improving the quality of junior doctor weekend handover. BMJ Qual Improv Rep 2014:2:u202379.w1297.

26 Bethune R, Campbell K, Rose A, et al. Improving weekend handover between junior doctors on medical and surgical wards. BMJ Qual Improv Rep 2014;2:u483.w1045.

27 Ward J, Spencer R, Soo E, et al. Standardising the organisation of clinical equipment on surgical wards at North Bristol NHS Trust: a quality improvement initiative. BMJ Qual Improv Rep 2015;4:u208308.w3441.

28 Tarrant M, Honeyman C, Aquilina A, et al. Improving the accessibility of trust guidelines for junior doctors at North Bristol NHS Trust. BMJ Qual Improv Rep 2014;3:u202211.w1101.

29 Andersson J, Bull T, Paul D, et al. Safer fluid prescribing at North Bristol Trust: Bringing practice in line with NICE Guidance with a redesign of the fluid prescription chart. BMJ Qual Improv Rep 2015;4:u203816.w1911.

30 Lyons N, Heron P, Bethune R. Improving the recording of surgical drain output. BMJ Qual Improv Rep 2015;4:u209264.w3964.

31 Patton M. Qualitative evaluation and research methods. Beverly Hills, CA: Sage 1990: 169-86

32 Braun V, Clarke V. Using thematic analysis in psychology. Qual Res Psychol 2006;3:77-101.

33 Research scan: Involving junior doctors in quality improvement. Report. Health foundation. 2011. Available from: http://www.health.org.uk/sites/default/files/Involvin gJuniorDoctorsInQualitylmprovement.pdf;

34 Russ SJ, Sevdalis N, Moorthy K, et al. A qualitative evaluation of the barriers and facilitators toward implementation of the WHO surgical safety checklist across hospitals in England: lessons from the "Surgical Checklist Implementation Project". Ann Surg 2015;261:81-91. 
35 Brewster L, Mountain G, Wessels B, et al. Factors affecting front line staff acceptance of telehealth technologies: a mixed-method systematic review. J Adv Nurs 2014;70:21-33.

36 de Silva D, 2015. Evidence Scan: What's getting in the way? Barriers to improvement in the NHS. Health Foundation. Available from: http://www.health.org.uk/publication/ what\%E2\%80\%99s-getting-way-barriers-improvement-nhs\#sthash.yOvv13sU.dpuf

37 Dixon-Woods M. The problem of context in quality improvement. London: Health Foundation, 2014

38 Aveling EL, McCulloch P, Dixon-Woods M. A qualitative study comparing experiences of the surgical safety checklist in hospitals in high-income and low-income countries. BMJ Open 2013;3:e003039.
39 Mackintosh N, Watson K, Rance S, et al. Value of a modified early obstetric warning system (MEOWS) in managing maternal complications in the peripartum period: an ethnographic study. BMJ Qual Saf 2014;23:26-34.

40 Kreindler SA. Six ways not to improve patient flow: a qualitative study. BMJ Qual Saf 2017;26:doi: 10.1136/bmjqs-2016-005438.[Epub ahead of print: 27 Jul 2016]

41 Roland D, Charadva C, Coats T, et al. Determining the effectiveness of educational interventions in paediatric emergency care. Emergency Medicine Journal 2014;31:787.3-8.

42 Institute for Healthcare Improvement Open School. 2018. Available from: http://www. ihi.org/education/ihiopenschool/Pages/default.aspx 